\title{
The Dynamical Masses, Densities, and Star Formation Scaling Relations of Lyman- $\alpha$ Galaxies
}

\author{
James E. Rhoads ${ }^{1}$, Sangeeta Malhotra ${ }^{1}$, Steven L. Finkelstein ${ }^{2}$, Johan P. U. Fynbo ${ }^{3}$, Emily \\ M. McLinden ${ }^{1,2}$, Mark L. A. Richardson ${ }^{1}$, Vithal S. Tilvi ${ }^{4}$
}

\begin{abstract}
We present the first dynamical mass measurements for Lyman- $\alpha$ galaxies at high redshift, based on velocity dispersion measurements from rest-frame optical emission lines and size measurements from HST imaging, for a sample of nine galaxies drawn from four surveys. These measurements enable us to study the nature of Lyman- $\alpha$ galaxies in the context of galaxy scaling relations. The resulting dynamical masses range from $10^{9}$ to $10^{10} M_{\odot}$. We also fit stellar population models to our sample, and use them to plot the Lyman- $\alpha$ sample on a stellar mass vs. line width relation. Overall, the Lyman- $\alpha$ galaxies follow well the scaling relation established by observing star forming galaxies at lower redshift (and without regard for Lyman- $\alpha$ emission), though in $\sim 1 / 3$ of the Lyman- $\alpha$ galaxies, lower-mass fits are also acceptable. In all cases, the dynamical masses agree with established stellar mass-linewidth relation. Using the dynamical masses as an upper limit on gas mass, we show that Lyman- $\alpha$ galaxies resemble starbursts (rather than "normal" galaxies) in the relation between gas mass surface density and star formation activity, in spite of relatively modest star formation rates. Finally, we examine the mass densities of these galaxies, and show that their future evolution likely requires dissipational ("wet") merging. In short, we find that Lyman- $\alpha$ galaxies are low mass cousins of larger starbursts.
\end{abstract}

Subject headings: galaxies: high-redshift — galaxies: formation — galaxies: evolution

\footnotetext{
${ }^{1}$ School of Earth and Space Exploration, Arizona State University, Tempe, AZ 85287; email James.Rhoads@asu.edu

2 Department of Astronomy, University of Texas at Austin, 1 University Station C1400, Austin, TX 78712, USA

${ }^{3}$ DARK Cosmology Centre, Niels Bohr Institute, University of Copenhagen, Juliane Maries Vej 30,2100 Copenhagen $\varnothing$, Denmark

${ }^{4}$ George P. and Cynthia W. Mitchell Institute for Fundamental Physics and Astronomy, Department of Physics, Texas A\&M University, College Station, TX 77843
} 


\section{Introduction}

Lyman $\alpha$ line emission is an increasingly important tool for identifying actively star forming galaxies in the distant universe. First proposed as a potential signpost for primitive galaxies (Partridge \& Peebles 1967), Lyman- $\alpha$ emission has now been used to identify thousands of galaxies at redshifts $2<z<7$, along with smaller samples at $z<2$ and several candidates at $z>7$.

While no galaxy yet identified by any means is demonstrably primordial, Lyman- $\alpha$ selected samples do have several properties suggestive of youth. Their starlight is dominated by young populations with characteristically low stellar masses (Pirzkal et al. 2007; Finkelstein et al. 2007) and small sizes (Bond et al. 2009; Malhotra et al. 2012). Yet, the correlation properties of these objects suggest that they are associated with moderately large halos (mass $\sim 10^{11} M_{\odot}$; Kovač et al. (2007); Guaita et al. (2010)). Combining these results suggests that a Lyman- $\alpha$ galaxy contains only a small fraction of the baryons that should be associated with its host dark matter halo. It would be interesting to know whether the "missing" baryons are present, either as old stellar populations, or in the interstellar medium of the Lyman- $\alpha$ galaxy.

Dynamical mass estimates for Lyman- $\alpha$ galaxies could potentially address this question, providing a standard for comparison with both the stellar masses and the dark halo masses. However, such dynamical mass estimates require an accurate measurement of the galaxy's velocity dispersion. The easiest approach to kinematics would be to use the Lyman- $\alpha$ line width, which is measured for most spectroscopically confirmed Lyman- $\alpha$ galaxies. Unfortunately, this does not lead to useful velocity dispersion information: The Lyman- $\alpha$ line profile can be dramatically affected by the interplay of resonant scattering and gas kinematics in the emitting galaxy.

We therefore turn in this paper to studying Lyman- $\alpha$ galaxy kinematics using the strong rest-frame optical emission lines of [OIII] $\lambda 5007 \AA$ and $\mathrm{H} \alpha \lambda 6561 \AA$. We build on the first detections of such lines in Lyman- $\alpha$ selected galaxies at redshifts $2.2 \lesssim z \lesssim 3.1$ (McLinden et al. 2011; Finkelstein et al. 2011; Hashimoto et al. 2012). In section 2, we describe the sample and the key data. In section 3 we estimate dynamical masses based on the observed line widths. In section 4, we analyze the stellar populations and dust reddening in these galaxies, using deep archival photometry. In section 5, we combine the rest-optical line width measurements with the stellar masses from population synthesis modelling to compare these Lyman- $\alpha$ galaxies with expectations from the stellar mass Tully-Fisher relation. In section 6 , we examine the relation between gas mass surface density and star formation surface density to show Lyman- $\alpha$ galaxies lie on the same sequence as starburst galaxies. Finally, in section 17, we explore the mass densities of the sample and the implications for the future 
evolution of Lyman- $\alpha$ galaxies.

Throughout the paper, we adopt a $\Lambda$-CDM "concordance cosmology" with $\Omega_{M}=0.27$, $\Omega_{\Lambda}=0.73$, and $H_{0}=71 \mathrm{~km} \mathrm{~s}^{-1} \mathrm{Mpc}^{-1}$.

\section{Description of Sample}

Our sample consists of Lyman- $\alpha$ emitting galaxies that were selected from four surveys. All are selected directly by the presence of a strong Lyman- $\alpha$ emission line in the survey data. For the analysis in this paper, we make use of (a) velocity dispersions $\sigma$, derived from spectra of rest frame optical emission lines; (b) sizes, as measured by the half-light radius $r_{e}$ in broad-band optical Hubble Space Telescope images; (c) stellar masses and dust extinctions, derived from spectral energy distribution (SED) fits; and (d) star formation rates (SFR), determined from $\mathrm{H} \alpha$ line flux measurements where available (and from spectral energy distribution fitting otherwise), and corrected for dust using the results of the SED fits. In this section we summarize the sources for various galaxies in the sample, along with the measurements of $\sigma, r_{e}$, and SFR. The stellar mass derivations are discussed later, in section 4. The properties of the sample are also summarized in tables 1-3.

\subsection{Bok telescope $z=3.1$ survey objects:}

First, we select two Lyman- $\alpha$ galaxies from a $5020 \AA$ narrowband survey using the Steward Observatory's 90 inch Bok Telescope on Kitt Peak, Arizona. The objects we study here were spectroscopically confirmed as Lyman- $\alpha$ emitters at $z \approx 3.12$ using the 6.5m MMT on Mt. Hopkins, Arizona. This survey was introduced in McLinden et al. (2011), and further details will be presented in a forthcoming paper (McLinden \& al 2013, in prep). We have followed up galaxies from this sample with three near-infrared spectrographs: LUCIFER, on the Large Binocular Telescope (McLinden et al. 2011); NIFS, on Gemini North (Richardson et al. 2013, in prep); and NIRSPEC, on the Keck II telescope (McLinden \& al 2013, in prep). The objects studied here are LAE40844 and LAE27878 (following the numbering in McLinden et al. (2011)).

The primary source of line widths for these objects is our Gemini NIFS data (Richardson et al. 2013, in prep), since our Keck observations of 40844 were primarily aimed at the (still undetected) [OII] $\lambda \lambda 3726,3729$ lines, while the kinematic line width is not well resolved in the LUCIFER observations. These Gemini observations yielded line widths of $\Delta V_{\mathrm{FWHM}}=13.8 \AA$ for object 40844 and $8.6 \AA$ for object 27878 . The instrumental resolution was about $5 \AA$. Sub- 
tracting this instrumental resolution in quadrature from the measured line widths yields $13.0 \AA$ and $7.0 \AA$ respectively. The corresponding line-of-sight velocity dispersions become $\sigma_{\mathrm{los}}=80 \mathrm{~km} \mathrm{~s}^{-1}$ and $\sigma_{\mathrm{los}}=43 \mathrm{~km} \mathrm{~s}^{-1}$ respectively.

The half-light radii for these objects are $1.1 \mathrm{kpc}$ for LAE40844 and $1.3 \mathrm{kpc}$ for LAE27878 (Malhotra et al. 2012). We estimated their star formation rates from SED fitting (section 4), since their $\mathrm{H} \alpha$ line falls at $2.70 \mu \mathrm{m}$ and cannot be easily observed. We obtained $120 M_{\odot} \mathrm{yr}^{-1}$ for LAE40844, and $34 M_{\odot} \mathrm{yr}^{-1}$ for LAE27878. We estimate the uncertainties in these star formation rates from the range of SFR in models yielding acceptable fits to the data (see section (4).

\subsection{HETDEX Pilot Survey objects:}

Second, we use two Lyman- $\alpha$ galaxies selected using a blind spectroscopic search with an integral field spectrograph as part of the HETDEX Pilot Survey. These are objects $\operatorname{HPS} 194(z=2.287)$ and $\operatorname{HPS} 256(z=2.491)$. For these, we base our kinematic line widths on Keck+NIRSPEC H $\alpha$ line measurements from Finkelstein et al. (2011); Song \& al (2013, in prep). These used low-resolution mode with the NSPEC-7 blocking filter. The measured line widths were $18.1 \pm 0.6 \AA$ (FWHM) for HPS 194, and $19.4 \pm 0.9 \AA$ (FWHM) for HPS 256. The instrumental resolving power is $R \sim 1500$ with the $0.76^{\prime \prime}$ slit, corresponding to

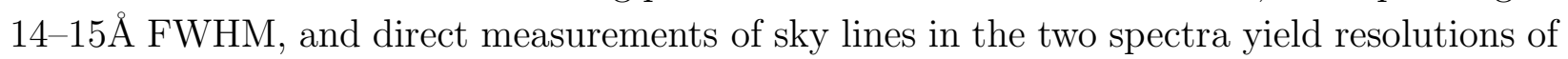
$14.4 \AA$ and $14.1 \AA$ (FWHM) respectively. Subtracting these in quadrature yields line widths of $11.0 \AA$ and $13.3 \AA$, respectively. The corresponding line-of-sight velocity dispersions become $\sigma_{\text {los }}=65 \mathrm{~km} \mathrm{~s}^{-1}$ and $\sigma_{\text {los }}=74 \mathrm{~km} \mathrm{~s}^{-1}$ respectively. The half-light radii, as reported in the COSMOS ACS i-band catalog (Leauthaud et al. 2007), are 1.5kpc for HPS194, and 1.1kpc for HPS256. (HPS 194 in fact corresponds to a pair of continuum sources separated by $\sim 0.5^{\prime \prime}$ in the COSMOS survey's HST+ACS images. We assume that the strong Lyman- $\alpha$ and $\mathrm{H} \alpha$ emission come from the brighter and more compact source. The other source has a $2.4 \mathrm{kpc}$ half-light radius, which would raise our dynamical mass for this object by about $60 \%$.)

The H $\alpha$ line fluxes (Song \& al 2013, in prep) yield star formation rates of $17 M_{\odot} \mathrm{yr}^{-1}$ and $20 M_{\odot} \mathrm{yr}^{-1}$, respectively, before dust correction. This is based on the conversion $\mathrm{SFR}=$ $4.6 \times 10^{-42}\left(L_{H \alpha} / \operatorname{erg~s}^{-1}\right) M_{\odot} \mathrm{yr}^{-1}$, which is appropriate for a Chabrier (2003) initial mass function [IMF] (Twite et al. 2012). The inferred SFR would be $1.2 \times$ greater for a Kroupa (2003) IMF, and $1.8 \times$ greater for the IMF assumed in Kennicutt (1998). We correct these star formation rates for dust extinction within the emitting galaxy. The uncertainty in the star formation rate is dominated by the uncertainty in this dust correction, which we estimate 
by considering the full range of extinction among models with $\chi^{2} \leq \chi_{\min }^{2}+1$, and further allowing the possibility that the extinction of $\mathrm{H} \alpha$ could exceed the extinction of continuum starlight by up to a factor of 2 .

\subsection{Subaru NB survey objects:}

Third, we use four Lyman- $\alpha$ galaxies from a narrowband survey described by Hashimoto et al. (2012) and Nakajima et al. (2012). These objects are drawn from two fields - COSMOS (objects COSMOS13636 and COSMOS30679) and the Chandra Deep Field South (objects CDFS3865 and CDFS6482). We obtained line widths for all sources by measuring the plotted FWHM of emission line profiles in figures 1, 2, and 5 of Hashimoto et al. (2012). In general, the lines in this sample appear marginally resolved. In our analysis we regard the line width measurements as upper limits where appropriate.

The COSMOS field spectra were from Keck + NIRSPEC spectroscopy. Here we obtained directly measured FWHM of 16.6 and 16.5A for COSMOS13636 and COSMOS30679, respectively. With a resolving power of $R=1500$, the instrumental resolution corresponds to $13.8 \AA$, which we subtract in quadrature to yield nominal velocity dispersions $\sigma=57 \mathrm{~km} \mathrm{~s}^{-1}$ and $\sigma=55 \mathrm{~km} \mathrm{~s}^{-1}$, respectively. In each case, the measurement remains consistent with a fairly broad range, $0<\sigma \lesssim 96 \mathrm{~km} \mathrm{~s}^{-1}$.

For these COSMOS field sources, we base the half light radii on the COSMOS ACS i-band catalog, obtaining $0.79 \mathrm{kpc}$ and $1.82 \mathrm{kpc}$ respectively. Finally, their star formation rates as inferred from their $\mathrm{H} \alpha$ fluxes are $8.7 M_{\odot} \mathrm{yr}^{-1}$ and $10.5 M_{\odot} \mathrm{yr}^{-1}$ respectively.

The CDFS field source spectra (Hashimoto et al. 2012) were obtained with Magellan + MMIRS, and have a somewhat lower resolving power $(R \approx 1120$, corresponding to $18 \AA)$. The measured FWHM are $23 \AA$ for CDFS3865 and $20 \AA$ for CDFS6482. Subtracting the instrumental resolution in quadrature yields velocity dispersions of $105 \mathrm{~km} \mathrm{~s}^{-1}$ and $42 \mathrm{~km} \mathrm{~s}^{-1}$, respectively, where again there is considerable uncertainty for the narrower, semi-resolved line (consistent with $0<\sigma<71 \mathrm{~km} \mathrm{~s}^{-1}$ ). The star formation rates for these sources, based on their $\mathrm{H} \alpha$ line fluxes, are $125 M_{\odot} \mathrm{yr}^{-1}$ and $31 M_{\odot} \mathrm{yr}^{-1}$ respectively.

For the physical sizes of the CDFS sources, we downloaded archival HST imaging from the GEMS survey (Rix et al. 2004) and measured the half light radii. We used the SExtractor (Bertin \& Arnouts 1996) half light radius, and as a consistency check also measured the fluxes in a series of circular apertures and interpolated the resulting photometric growth curve. Both methods gave consistent answers, with half light radii of $0.96 \mathrm{kpc}$ for CDFS3865, and $1.78 \mathrm{kpc}$ for CDFS6482. 


\section{4. $\quad \mathrm{ESO} z=2.25$ survey object:}

Finally, we use one Lyman- $\alpha$ galaxy, LAE-COSMOS-47, from a narrowband-selected $z=2.25$ COSMOS field sample obtained by Nilsson et al. (2011) using the ESO 2.2m telescope, with followup observations obtained by Fynbo and collaborators using the X-Shooter

spectrograph (Vernet et al. 2011) on the VLT. The X-Shooter spectrum provided a well constrained velocity dispersion measurement of $30 \mathrm{~km} \mathrm{~s}^{-1}$. The star formation rate, derived from the $\mathrm{H} \alpha$ line flux, is $33 M_{\odot} \mathrm{yr}^{-1}$. The half-light radius, from the COSMOS ACS catalog (Leauthaud et al. 2007), is $1.14 \mathrm{kpc}$.

\section{Dynamical Mass Estimates}

The measured velocity dispersions $\sigma$ from the rest-optical emission lines are a good estimate of the total luminosity-weighted kinematics of the gas. The galaxies we are studying are spatially unresolved even in $\sim 0.5^{\prime \prime}$ seeing, meaning that the ground-based spectra we use effectively sample the integrated light, with no important dependence on slit width.

The precise conversion from velocity width to mass will depend on the kinematic structure of these galaxies. For a pure rotation-supported model with a flat rotation curve, we expect $\sigma^{2}=\sin ^{2} i v_{c}^{2} / 2$, where $i$ is the inclination angle of the disk (with $i=90^{\circ}$ corresponding to an edge-on system).

The simplest dynamical mass estimate from these measurements, which is a lower bound to the true gravitating mass, is $M_{d y n} \geq v_{c}^{2} r / G$, where $r$ is the maximum radius at which we observe light from the galaxy. A more practical choice of radius is the effective radius $r_{e}$, defined as the radius that encloses half of the galaxy's light in projection. If we presume that the half-light radius is also the half-mass radius, our revised estimate of the dynamical mass becomes $M_{d y n} \approx 2 v_{c}\left(r_{e}\right)^{2} r_{e} / G \approx 4 \sigma^{2} r_{e} /\left(G \sin ^{2} i\right) \gtrsim 4 \sigma^{2} r_{e} / G$. The resulting mass estimates range from $10^{9}$ to $10^{10} M_{\odot}$, and are summarized in table 8 ,

Several circumstances could affect our estimated mass. A rotating disk with $i<90^{\circ}$ would reduce the measured $\sigma$ (relative to the edge-on case). Also, in our spatially unresolved spectroscopy, the observed $\sigma^{2}$ reflects the luminosity-weighted average kinematics. If a significant fraction of the galaxy's light is emitted from regions where the local circular speed $v_{c}(r)$ is below the maximum circular speed $\left(v_{\max }\right)$, we should expect the weighted average $\sigma^{2}$ to underestimate $v_{\max }$ and hence the mass. The precise magnitude of this effect depends on the galaxy's light profile and rotation curve. Also, like any dynamical mass based on luminous tracers, our estimate is insensitive to mass located outside the luminous matter distribution of the galaxies. If the galaxies are embedded in extended dark matter halos, the 
total mass of the halo could be many times the mass estimates derived from the observed $r_{e}$ and $\sigma^{2}$.

Turbulence in the galaxy's gas would contribute to the measured $\sigma$, though in virial equilibrium, that turbulence would constitute a source of pressure support and the ordered rotation of the galaxy would be correspondingly reduced. Most interestingly, if the galaxy is not in equilibrium at all, our assumed relations between kinematics and mass could be substantially wrong. Our mass estimate implicitly assumes that the virial theorem is fulfilled, that is, that the kinetic energy $K$ and potential energy $U$ of the galaxy are related by $K=-U / 2$. On the other hand, in a cold accretion scenario, new material falling into a galaxy for the first time should have $K=-U$, i.e., the motions are faster for the same gravitating mass under these conditions, and the mass inferred from gas motions would be correspondingly over-estimated by a factor up to $\sim 2$.

Given these uncertainties, it is best to regard our direct dynamical mass estimates as approximate numbers, good to a factor of perhaps 2 when regarded as lower bounds to the true dynamical mass. Other dynamical mass estimates in the literature consider a more general scaling coefficient so that $M_{d y n}=\beta \sigma^{2} r_{e} / G$ (see Toft et al. (2012) and references therein, esp. Jorgensen et al. (1996) and Cappellari et al. (2006)). These works favor $\beta \approx 5$ for early-type galaxies with Sersic index $n \approx 4$, and find that despite theoretical expectations for some increase of $\beta$ with decreasing Sersic $n$, the observational evidence favors $\beta \approx 5$ for a wide range of $n$. Thus, the simple arguments that led us to use $\beta=4$ likely come fairly close to the correct dynamical masses.

A complementary approach to interpreting the kinematic data on these galaxies is to use their linewidths to place them on some form of the Tully-Fisher relation, and so to compare them on an equal footing to other galaxy populations. Such an approch avoids the difficulties associated with identifying the right radius to use in estimators of the form $M \sim v^{2} R / G$. For high redshift galaxy populations, a small scatter with weak redshift evolution has been demonstrated for the stellar mass Tully-Fisher relation, and we place our galaxies on such a relation in section 5 below. To do so, we first need their stellar masses.

\section{Population Synthesis Modeling}

All of the galaxies we study have extensive multiband photometry in the literature, generally including multiband optical data, some deep ground-based near-infrared photometry, and Spitzer IRAC (Fazio et al. 2004) observations that are deep enough to be constraining in at least the $3.6 \mu m$ channel. 
We have used these data to derive stellar mass estimates for the full sample. Stellar mass estimates are also available in the published literature for many of these galaxies (Finkelstein et al. 2011; Hashimoto et al. 2012; Nakajima et al. 2012; McLinden \& al 2013, in prep). While these are mostly consistent with our estimates where samples overlap, we opted to fit the entire sample using a single procedure to avoid potential difficulties comparing masses derived using different methodologies.

\subsection{General comments on SED fitting:}

(1) The strong Lyman- $\alpha$ emission in these objects requires the presence of a young stellar population, whose ultraviolet light ionizes interstellar hydrogen that then recombines to produce the observed Lyman- $\alpha$ radiation. (2) The amount of dust in the fitting is essentially determined by the UV spectral slope (cf. Meurer et al. (1997); Hathi et al. (2008); Finkelstein et al. (2011)). (3) In some objects, no stars older than $10^{7}$ years are required to explain the observed light. (4) A considerable mass in old stars is permitted. (5) The ionizing photon production for the best-fit stellar populations can be converted to a Lyman- $\alpha$ luminosity by assuming that $2 / 3$ of the ionizing photons are ultimately converted to Lyman- $\alpha$ radiation (as expected under Case B recombination with a negligible escape fraction for ionizing photons). Combining this line luminosity estimate with the directly measured Lyman- $\alpha$ line flux gives an estimate of the escape fraction for the Lyman- $\alpha$ photons. These escape fractions are sensitive to the details of the star formation history over the last $\sim 6$ Myr. We can say with reasonable confidence that the resulting escape fraction is of order half for the most plausible models, and that Lyman- $\alpha$ escape fractions below 20-30\% are ruled out unless we change the stellar population in some way that dramatically increases the ionizing photon production. (6) The estimated stellar population ages and masses would increase dramatically if we did not account for the [OIII] $\lambda \lambda 4959,5007$ line fluxes. In this case, the stellar population fitting code attempts to interpret the red $\mathrm{H}-\mathrm{K}$ color and the bright flux in the rest-frame $5000 \AA$ range as due to older stellar populations. This effect can exceed an order of magnitude in both age and mass when the filter containing the [OIII] $\lambda \lambda 4959,5007$ lines is the reddest filter considered, while it is usually smaller when an additional filter redward of the $4000 \AA$ break is included (meaning, in our case, the IRAC photometry).

\subsection{Starburst99 modeling:}

We measured stellar masses for our sample using the Starburst99 population synthesis code (Leitherer et al. 1999). For each galaxy we started with publically available broad 
band photometry. For the seven objects in the COSMOS field, we used COSMOS project broad band photometry (Capak et al. 2007; McCracken et al. 2010), either directly from the COSMOS archive, or as quoted in the papers defining the samples (Finkelstein et al. 2011; Hashimoto et al. 2012; Nakajima et al. 2012). For the two CDFS objects, we used MUSYC survey photometry (Gawiser et al. 2006) (again as quoted in Hashimoto et al. (2012); Nakajima et al. (2012)). In all cases, our final model fitting used at least 9 photometric bands spanning at least the wavelength range $0.4 \mu m<\lambda<3.6 \mu m$.

To interpret the photometry in terms of stellar population parameters, we first ran Starburst99 to generate a grid of model spectra for stellar populations at a range of ages, from $2 \times 10^{6}$ years (which is so young that no star has yet left the main sequence), up to $2 \times 10^{9}$ years (which is the age of the universe at $z \approx 3.1$ ), and using a Kroupa (2003) IMF. We then assumed a star formation history (as described below) and generated a model spectrum by a linear combination of the spectra for particular age steps from the Starburst99 output.

We account for the mean opacity of the intergalactic medium using the prescription of Madau (1995). We do not treat the variance in the IGM opacity in the present work. (Doing so would effectively add uncertainty to the expected fluxes in the $u^{*}, \mathrm{~B}_{j}$, and $g$ bands, and so would improve the model $\chi^{2}$, but would not likely change the best fit model parameters much.)

We model dust in our sample galaxies using the Small Magellanic Cloud (SMC) extinction law from Pei (1992), and treating the extinction as a thin screen. The SMC law is a reasonable choice since its metallicity corresponds approximately to the few metallicity constraints so far available for Lyman- $\alpha$ emitters (Finkelstein et al. 2011).

We next added the directly measured spectroscopic line fluxes to the model spectrum at the appropriate wavelengths, since these emission lines are not included in the model output. This step follows the application of IGM and dust opacity, since nature has already applied these effects to the observed line fluxes.

At this point, we have a full model spectrum accounting for stellar populations, emission lines, dust, and intergalactic hydrogen absorption. We multiply this spectrum by the bandpass of each filter in the photometric data set, integrate, and normalize appropriately to obtain model fluxes in each observed filter for the model under consideration. These can be compared to the observed data to obtain a goodness-of-fit parameter $\chi^{2}=$ $\sum_{j=1}^{N}\left(f_{j, o b s}-f_{j, m o d}\right)^{2} /\left(\delta f_{j, o b s}\right)^{2}$.

To optimize the model, we fitted the observed spectral energy distribution of the sample galaxies by a simple Monte Carlo approach that randomly varies the amount of dust and 
the mass in stars in each of 13 logarithmically spaced age bins, and accepts a change to the model parameters using $\chi^{2}$ minimization. This approach allows a more general star formation history than a single burst or an exponentially decaying star formation rate. While the resulting sampling of stellar ages is somewhat coarse compared to a typical single burst model, the associated uncertainty is not a dominant factor in our stellar mass estimates. The model fits usually converge to a case where 1-3 of the 13 mass bins dominate the luminosity at all observed wavebands. We therefore "trim" the parameter list by fixing the stellar mass to zero in bins that are clearly of minor importance, and rerun the fit with only the important mass bins. This "trimming" step generally has a negligible effect on the final $\chi^{2}$, confirming that 1-3 simple stellar populations can explain the observed spectrum as well as a more complex star formation history.

To further explore the parameter space of acceptable fits, we modified our code to optimize for either minima or maxima of either stellar mass or dust extinction, subject to the constraint that the model $\chi^{2}$ remain close to the $\chi^{2}$ of the best fitting model for each object. We explored models with $\Delta \chi^{2}=+1$ and $\Delta \chi^{2}=+4$. This corresponds approximately to the $1 \sigma$ and $2 \sigma$ error regions in the parameter space.

\section{The Stellar Mass Tully-Fisher Relation}

The stellar mass Tully-Fisher (SMTF) relation is a correlation between the kinematic line widths of galaxies and their stellar masses. The SMTF relation is more robust to differences in stellar population mass-to-light ratio than the original Tully-Fisher relation (which correlates luminosity with line width; Tully \& Fisher (1977)). The relation is further generalized by Kassin et al. (2007), who demonstrated that replacing the circular speed $V_{c}$ with the kinematic estimator $S_{0.5}=\left(0.5 V_{\text {rot }}^{2}+\sigma^{2}\right)^{1 / 2}$ results in an SMTF that is both tighter and more applicable to the wide range of galaxy properties seen at high redshift. $S_{0.5}$ has an additional advantage: For spatially unresolved galaxies (like those we study here), $S_{0.5}$ can be measured reasonably accurately regardless of whether the width is dominated by ordered rotation or by random motions.

Our actual measurement is a single number, the line width, characterized by the observed line-of-sight velocity dispersion $\sigma_{o b s}$. We then use $S_{0.5}=\sigma_{o b s}$ for our galaxies. Consider pure circular motion with a flat rotation curve of velocity $V_{c}$, viewed edge-on: we will find $\sigma_{o b s}^{2}=\left\langle\left(\sin (\phi) V_{c}\right)^{2}\right\rangle=0.5 V_{c}^{2}$, so that $S_{0.5}=\sigma_{o b s}$. If instead the motion is entirely random, with $V_{\text {rot }}=0$, we expect $\sigma_{o b s}=\sigma$, and again, $\sigma_{o b s}=S_{0.5}$. Only for ordered rotation in a face-on configuration do we expect $\sigma_{o b s}$ to be a significant under-estimate of $S_{0.5}$. While we cannot rule out this possiblity with our data, it is likely that these galaxies are dynamically 
hot $\left(\sigma / V_{\text {rot }} \nless 1\right)$, since the stellar populations dominating the observed light are at most $\sim 1$ dynamical time old.

Our results are shown in figure 1, both for stellar masses from SED fitting and for dynamical masses from line width and spatial extent. The error bars for the stellar masses show the ranges of stellar mass permitted by models with $\Delta \chi^{2} \equiv \chi^{2}-\chi_{\min }^{2}<4$, and with $\Delta \chi^{2} \leq 1$. The error bars for dynamical mass are determined by the uncertainties in $\sigma$, and are diagonal since the $\mathrm{x}$-axis of the plot is $\sigma$. For three objects from the Hashimoto et al. (2012) sample, $\sigma$ and $M_{d y n}$ are plotted as upper bounds.

Comparing our Lyman- $\alpha$ sample to the stellar mass Tully-Fisher relation that Kassin et al. (2007) reported for $0.1<z<1.2$ star-forming galaxies, we find that both the stellar masses and dynamical masses of LAEs generally follow the established relation. Dynamical mass estimates show less scatter than stellar masses, as might be expected given the vagaries of star formation histories.

About half the galaxies are consistent with stellar masses falling a factor of two or more below their dynamical masses, when we account for the range of acceptable estimates for both. For these galaxies, it is possible that the dynamical mass within the central $1-2 \mathrm{kpc}$ is dominated not by the young stars that power the observed Lyman- $\alpha$ emission, but by some other unseen component. Collisionless dark matter should not be so strongly concentrated in the central kpc of a dark matter halo. Old stars (formed from centrally concentrated gas $\gg 10^{8}$ years ago) are a possible alternative. The dynamical mass estimates we present are in fact a tighter limit on the total mass in old stars within the inner $\sim$ kpc than are the photometric limits. Given the actively star-forming nature of these Lyman- $\alpha$ emitting galaxies, a reservoir of gas is the most intriguing possibility for the "excess" dynamical mass. Overall, though, such excess mass is merely permitted and not required by the dataso, overall, the Lyman- $\alpha$ galaxy sample shows consistency with the stellar mass Tully-Fisher relation for other samples. Whatever physical properties allow Lyman- $\alpha$ to escape these particular star forming galaxies, they do not strongly affect the mass-linewidth relation.

The tight correlation observed between $M_{d y n}$ and line width is related to the small and nearly constant physical sizes of the Lyman- $\alpha$ galaxy sample (Malhotra et al. 2012). A fixed physical size, combined with variable (and sometimes uncertain) line widths, can generate the observed slope of the $M_{d y n}-S_{0.5}$ relation. The $M_{\star}-S_{0.5}$ relation from Kassin et al. (2007) has a somewhat steeper slope. Presuming that stars form a fairly large and fairly constant fraction of the dynamical mass, this slope could be interpreted as evidence for size-linewidth relation in the Kassin et al. (2007) sample, with $r_{e} \propto S_{0.5}^{\gamma}$ for $\gamma \sim 1$. 


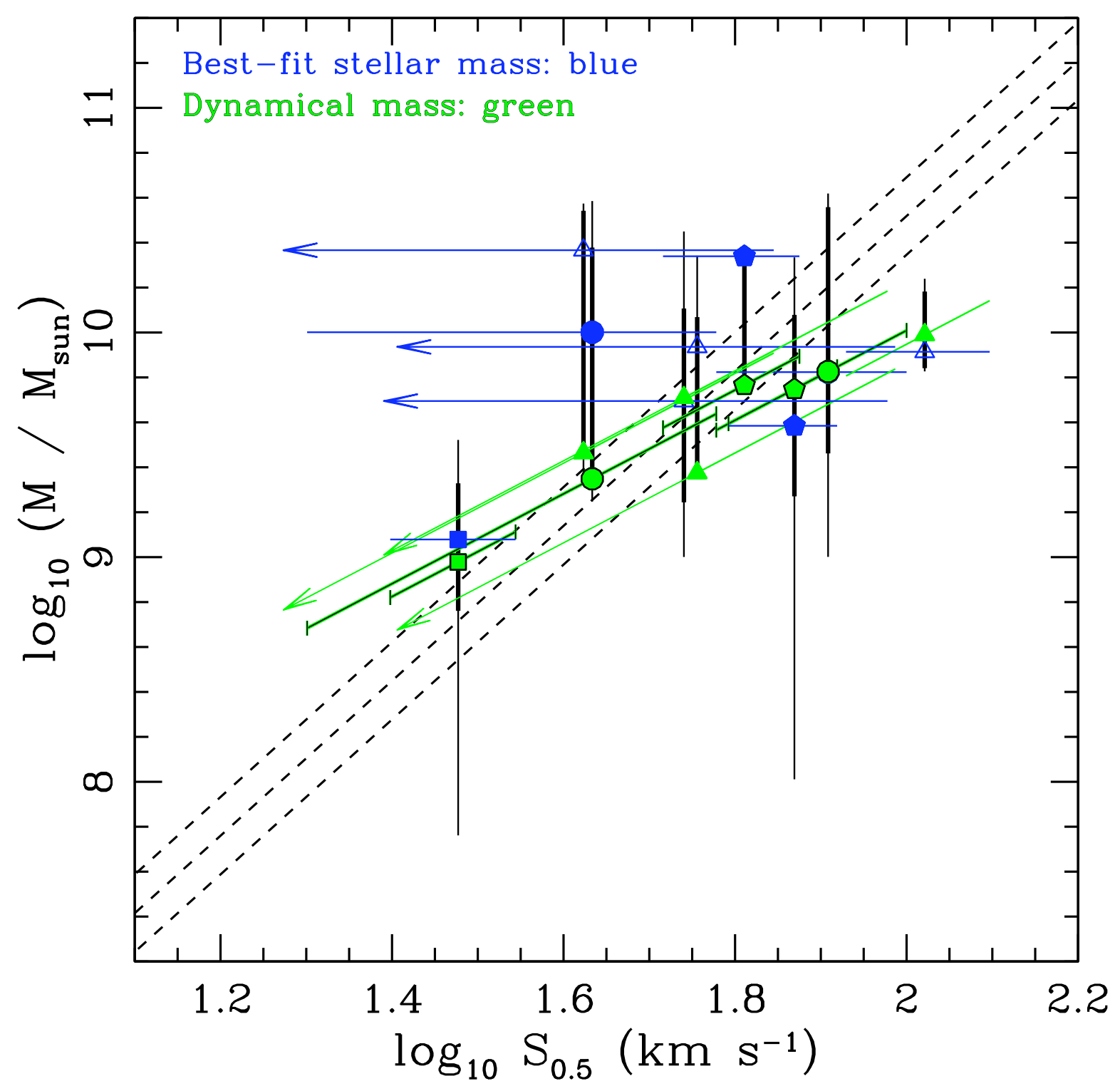

Fig. 1.- Stellar and dynamical mass Tully-Fisher relation. Colored points mark the Lyman$\alpha$ emitting galaxies for which we present dynamical mass estimates. For each, we plot the best fitting stellar mass (blue) and the dynamical mass (green). The vertical bar through each point-pair spans the range of stellar masses for acceptable models (thicker line: models with $\Delta \chi^{2}<1$; thinner line: models with $1<\Delta \chi^{2}<4$ ). The error bars on dyamical masses are diagonal, since uncertainty in velocity dispersion affects both plotted quantities. Point shapes correspond to surveys: Circles $\leftrightarrow$ Bok telescope $z=3.1$ survey; pentagons $\leftrightarrow$ HETDEX pilot survey; triangles $\leftrightarrow$ Hashimoto et al. (2012) Subaru survey; and square $\leftrightarrow$ Nilsson et al. (2011) survey. Objects with marginally resolved lines are plotted using their best-estimate values of $\sigma$, but can be regarded as upper limits on both $S_{0.5}$ and $M_{d y n}$. Dashed lines are the best-fit stellar mass Tully-Fisher relation from Kassin et al. (2007). 


\section{Star formation scaling laws}

We now take the dynamical mass as an upper bound on the gas mass in these galaxies, and compare their properties to the scaling relations that describe star formation in other galaxy classes.

We use the gas surface mass density limit $\Sigma_{g, \max }=M_{d y n} /\left(2 \pi r_{e}^{2}\right)$. We can improve this bound by subtracting our stellar mass estimates. In practice, our maximum stellar mass always exceeds the dynamical mass, allowing the possiblity that there is no gas mass. In most cases, though, our minimum stellar masses are below the dynamical masses, and we can subtract them to yield refined estimates of the gas mass surface density $\left(\Sigma_{g} \lesssim\right.$ $\left.\left(M_{d y n}-M_{\star, \min }\right) /\left(2 \pi r_{e}^{2}\right)\right)$.

The star formation rate and gas surface density can be related according to scaling laws of the form $\log \Sigma_{S F R} \approx \alpha_{S F} \log \Sigma_{g}+\beta_{S F}$ (where $\Sigma_{S F R}$ is in $M_{\odot} \mathrm{yr}^{-1} \mathrm{kpc}^{-2}$, and $\Sigma_{g}$ is in $\left.M_{\odot} \mathrm{kpc}^{-2}\right)$. For nearby spirals, and for distant star-forming BzK galaxies, Daddi et al. (2010) find $\alpha_{S F}=1.42$ and $\beta_{S F}=-9.83$ for our choice of units. For submillimeter galaxies and (U)LIRGS, Daddi et al find a parallel but offset "starburst" sequence, with $\beta_{S F} \approx-8.93$ (corresponding to $8 \times$ more star formation for the same gas surface density).

We determined $\Sigma_{S F R}$ for our sample using the SFR estimates and half-light radius measurements discussed in 2. We have selected a Chabrier IMF for consistency with Daddi et al. (2010). These values of $\Sigma_{S F R}$ exceed the expectations for "normal" star forming galaxies by a median factor of 4 , based on $\Sigma_{g, \max }$ alone (i.e. assuming that all the gravitating mass is gas). The disagreement is significant at the $>3 \sigma$ level, relative to the 0.33 dex scatter in the scaling relation reported by Daddi et al. (2010). A factor of 4 would place the Lyman- $\alpha$ galaxies in between the normal and starburst sequences, though closer to the starburst sequence. If we use $\Sigma_{g}=\left(M_{d y n}-M_{\star, \min }\right) /\left(2 \pi r_{e}^{2}\right)$ in the scaling relations, we find that the median galaxy in our sample is forming stars at twice the rate expected even under the starburst scaling. The bottom line from this comparison is that Lyman- $\alpha$ galaxies likely belong to a family of starbursting objects that includes ULIRGS and submillimeter galaxies, despite order-of-magnitude differences in mass and star formation rates.

While the "normal" and starburst galaxies follow distinct $\Sigma_{S F R}-\sigma_{g}$ relations, they obey a single relation when $\Sigma_{g}$ is replaced with the quantity $\Sigma_{g} / \tau_{d y n}$, where $\tau_{d y n}$ is the dynamical time. We use $\tau_{d y n} \approx 2 \pi r_{e} / \sigma$ to place our Lyman- $\alpha$ galaxy sample on this relation also. While the Lyman- $\alpha$ galaxies appear less unusual when measured against this relation, they remain systematically above the trend line found by Daddi et al. (2010). The difference is suggestive, rather than significant, being a $2 \sigma$ effect. The median offset is a factor of $\sim 3$ in

$\Sigma_{S F R}$ at fixed $\Sigma_{g} / \tau_{d y n}$. This is comparable to the $0.44 d e x$ scatter in the relation as reported 

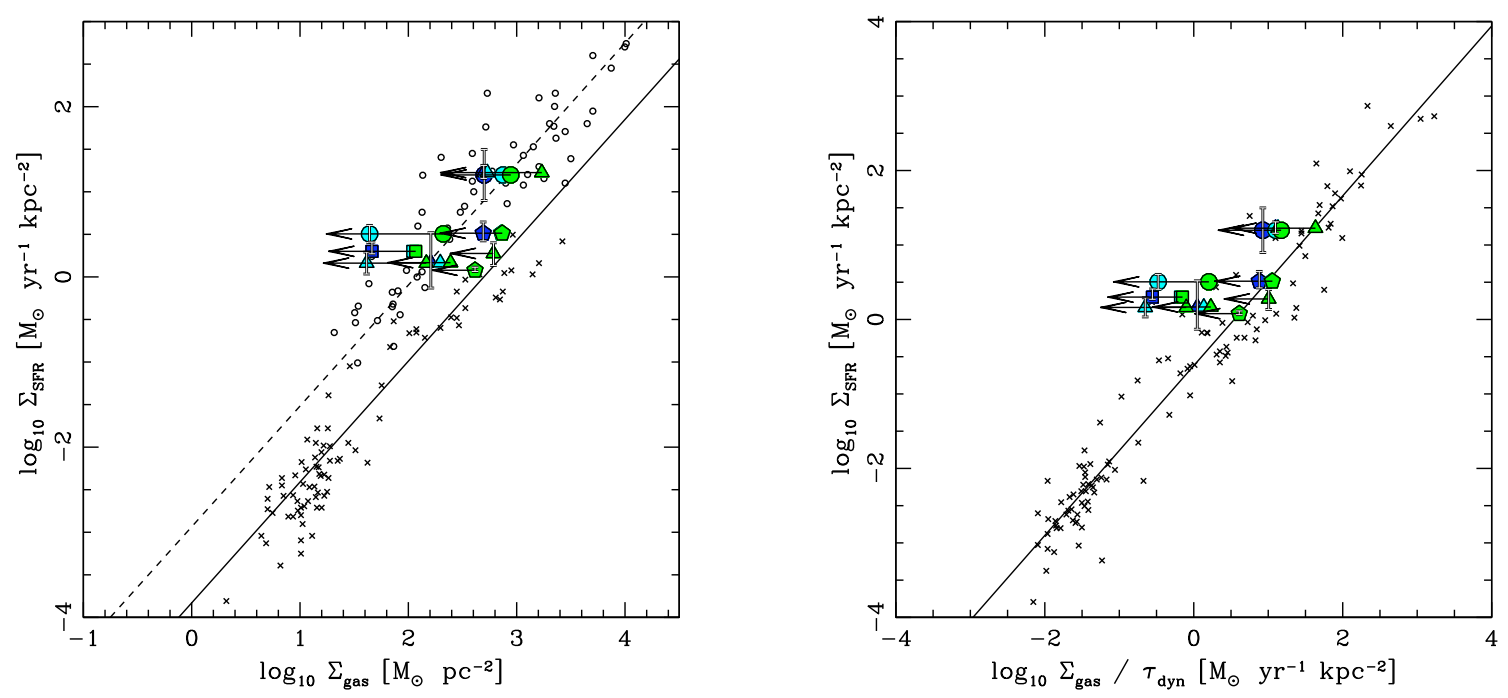

Fig. 2.- Star formation law comparison. First panel: Relation between star formation rate surface density $\Sigma_{S F R}$ and gas mass surface density $\Sigma_{g}$, both for our samples (large colored points) and for comparison samples drawn from Daddi et al. (2010). For each Lyman- $\alpha$ galaxy, the green point marks the upper bound on gas surface density obtained by associating the entire dynamical mass with gas. Cyan and blue points take a portion of the dynamical mass to be associated with stars, using the " $2 \sigma$ " and " $1 \sigma$ " low-mass models from out stellar population fitting. Error bars in $\Sigma_{S F R}$ account both for uncertainties in $\mathrm{H} \alpha$ flux and in extinction corrections. Point styles identify subsamples, as in figure 1, The solid line marks the relation for normal star forming galaxies, and " $\mathrm{x}$ " points the galaxy samples obeying that relation. The dashed line marks the starburst relation, and open black circles mark the corresponding galaxies. The Lyman- $\alpha$ galaxies are inconsistent with the normal star formation relation, and consistent with the starburst galaxy sequence. Second panel: $\Sigma_{S F R}$ vs. $\Sigma_{g} / \tau_{d y n}$. Since Daddi et al. (2010) report a single relation here, all points from that paper have the same style. The Lyman- $\alpha$ galaxies are consistent with the general relation, though higher at an insignificant but intriguing level $(\sim 2 \sigma)$. 
by Daddi et al. (2010)). This possible deviation should be explored using a larger sample.

The range of gas surface densities plotted in figure 2 is from $\sim 30$ to $\sim 1000 M_{\odot} \mathrm{pc}^{-2}$. Based on a standard ratio of dust to gas column density, $A_{B} \approx \Sigma_{g} /\left(11 M_{\odot} \mathrm{pc}^{-2}\right)$ (Bohlin et al. 1978), this corresponds to $A_{B} \sim 3-90$ magnitudes of extinction. Yet, our SED fits suggest modest extinctions, $A_{v} \lesssim 1$, in all cases. There are a few possible explanations. First, the gas surface density could be much lower than one would expect for the observed level of star formation activity. In this case, the star formation vs. gas surface density scaling must be more extreme than even the starburst relation. Second, the dust-to-gas ratio could be about 1-2 orders of magnitude lower than in the Milky Way. This would be most easily accommodated if the dust-to-gas ratio scales as the square of metal abundance. Third, the extinctions inferred from SED fitting could be dramatic under-estimates. If so, these objects' bolometric luminosity would mostly emerge in the rest-frame far infrared, making them readily detectible with submillimeter imaging (cf. Finkelstein et al. (2009)).

\section{Densities}

Given our estimates of the dynamical mass, it is straightforward to determine the mean density within the effective radius for our sample. We find

$$
\bar{\rho}_{e}=\frac{3}{2 \pi G} \frac{\sigma^{2}}{r_{e}^{2}}=\frac{3 \pi}{G t_{o r b}^{2}}
$$

where we have used $v_{c}=\sqrt{2} \sigma$ and $t_{\text {orb }}=2 \pi r_{e} / v_{c}$.

Hierarchical structure formation models suggest that most collapsed galaxies at $z \sim 3$ should be incorporated into early-type galaxies or disk galaxy bulges by the present epoch. We therefore compare the density measurements for Lyman- $\alpha$ galaxies to corresponding densities for early-type galaxies and bulges in figure 3. The Lyman- $\alpha$ galaxy densities are the mean density within the effective radius, derived dynamically and therefore inclusive of all gravitating mass. For disk galaxy bulges, we take an intermediate-redshift sample from MacArthur et al. (2008). Here we use the published effective radii and velocity dispersions to obtain dynamical estimates of density. We use two nearby elliptical galaxy samples (one from Kelson et al. (2000), and one compiled by Bezanson et al. (2009) from earlier work by Franx et al. (1989); Peletier et al. (1990); Jedrzejewski (1987)). For the Kelson et al. (2000) sample, we determine densities from published $\sigma$ and $r_{e}$ measurements; while for the Bezanson et al. (2009) sample, we use plotted values of $r_{e}$ and $\bar{\rho}\left(r_{e}\right)$. The Lyman- $\alpha$ galaxies are smaller at fixed density, and less dense at fixed radius, than are the nearby ellipticals and spiral bulges. On the other hand they tend to be smaller and denser than the handful 
of local ellipticals that have similar dynamical masses. We also compare to a sample of early type galaxies at $z \sim 2.3$ (Bezanson et al. 2009), which overlaps our Lyman- $\alpha$ sample in redshift. Here, the Lyman- $\alpha$ galaxies are of considerably lower density. In the samples from Bezanson et al. (2009), we are using stellar mass rather than dynamical mass, since $\sigma$ is generally unavailable for the high-redshift early type galaxies. Were dynamical masses available, we might expect these two samples to shift upwards in figure 3, but the effect would be modest, since the central regions of early type galaxies are likely dominated by mass in stars.

The relation between initial overdensity and epoch of collapse ensures that bound galaxies at $2<z \lesssim 3$ will, by the present epoch, be parts of more massive structures. We expect that the Lyman- $\alpha$ galaxies we observe will grow through some combination of merging and smooth accretion. Merging can proceed either without dissipation, as expected for pure mergers of stellar systems ("dry mergers"); or with dissipation, as generally expected for gas-rich objects. Conservation of energy and the virial theorem can be combined to infer the expected evolution of radius with mass for dry mergers, both in the limit of equal-mass ("major") mergers, and in the limit of large mass ratio ("minor") mergers. For major mergers, we expect $R_{e} \propto M$, while for minor, we expect $R_{e} \propto M^{2}$ (e.g., Bezanson et al. 2009). In figure 3, these correspond to $\rho \propto R_{e}^{-2}$ for major mergers, and $\rho \propto R_{e}^{-2.5}$ for minor. We plot vectors for both scalings. These show that the Lyman- $\alpha$ galaxies we observe cannot grow to reproduce the observed masses, sizes, and densities of modern elliptical galaxies through simple dry merging. Instead, dissipational ("wet") merging is required, in order to grow the galaxies at approximately constant mass density.

\section{Conclusions}

We have performed the first study of dynamical masses for high redshift Lyman- $\alpha$ galaxies, using a sample of nine objects assembled from four samples (McLinden et al. 2011; Richardson et al. 2013, in prep; Finkelstein et al. 2011; Hashimoto et al. 2012; Nakajima et al. 2012; Nilsson et al. 2011). Such studies have been previously impractical, given small sizes that require HST imaging for size measurements (Malhotra et al. 2012), faint continuum that precludes absorption line measurements, and redshifts that require near-infrared spectroscopy to access those emission lines most useful for kinematic line width measurements. We measure dynamical masses ranging from $10^{9} M_{\odot}$ to $10^{10} M_{\odot}$.

We combine these dynamical masses with stellar masses, to study the position of Lyman$\alpha$ galaxies on a version of the stellar mass Tully-Fisher relation between mass and line width. To derive stellar masses consistently for the full sample, we fitted population models to 


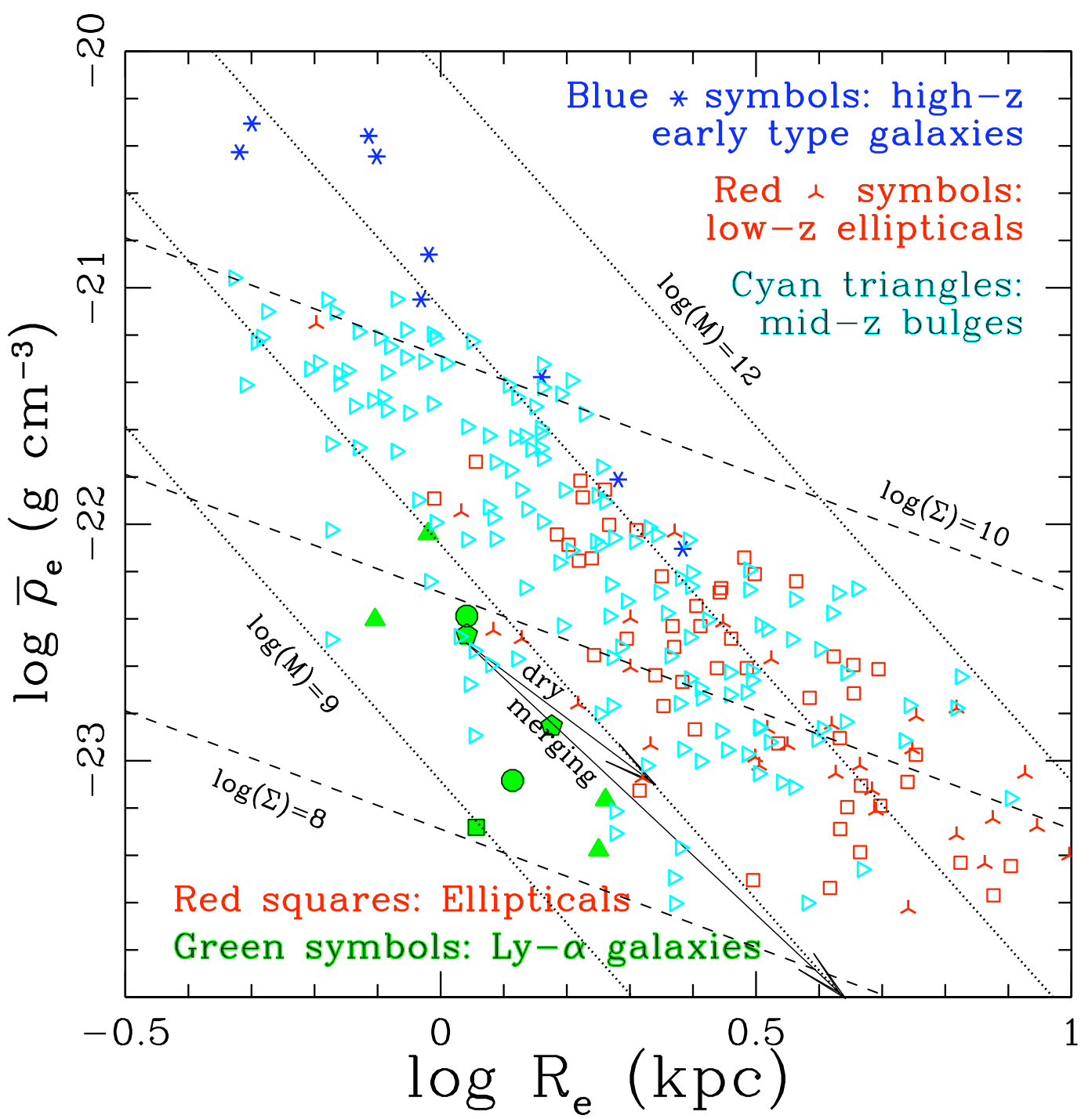

Fig. 3.- Densities and effective radii for Lyman- $\alpha$ galaxies (green circles) and comparison samples. Cyan triangles are intermediate-redshift disk galaxy bulges MacArthur et al. (2008), and red squares are low-redshift elliptical galaxies from Kelson et al. (2000), both with densities derived dynamically. Blue asterisks are high-redshift $(z \sim 2.3)$ early-type galaxies, and three-pointed stars are another local early type galaxy sample, both from Bezanson et al. (2009), and both using stellar masses estimates from SED fitting. Diagonal dotted lines mark masses of $10^{9}, 10^{10}, 10^{11}$, and $10^{12} M_{\odot}$, and dashed lines mark surface densities of $10^{8}, 10^{9}$, and $10^{10} M_{\odot} \mathrm{kpc}^{-2}$. The Lyman- $\alpha$ galaxies are typically about $10 \times$ less massive than the local ellipticals, yet of comparable density. Non-dissipative ("dry") merging results in galaxy densities that decrease as $\rho \propto R_{e}^{k}$ for $2 \leq k \leq 2.5$, based on virial theorem arguments and conservation of total energy (see, e.g., Bezanson et al. (2009)). Arrows show such scalings, with the shorter arrow corresponding to one major merger and the longer to a mass doubling through minor mergers. Neither arrow approaches the region of the elliptical galaxy samples, indicating that dissipational merging is required if these objects are indeed progenitors of present day early type galaxies. 
extensive multiband photometry covering rest wavelengths from Lyman- $\alpha$ to beyond the $4000 \AA$ break. This affords constraints on young stars, older stars, and dust.

We find that the Lyman- $\alpha$ galaxies are broadly consistent with the stellar mass TullyFisher relation established at lower redshift. Thus, whatever physical conditions allow the production and escape of significant Lyman- $\alpha$, they do not result in strong departures from the linewidth-mass relation. On the other hand, about half the sample galaxies are consistent with stellar masses significantly below their dynamical masses. In these cases, there is a possibility of a dynamically significant reservoir of gas that is present in the inner kpc region and provides fuel for ongoing star formation. The dynamical mass measurements are in fact the most powerful constraint we have on the presence of old stars in the sample galaxies. In most cases, the stellar population fits allow up to $\sim 3 \times 10^{10} M_{\odot}$ of old stars before the resulting light appreciably degrades the quality of the SED fit. However, dynamical constraints place a tighter limit of $\leq 1 \times 10^{10} M_{\odot}$ on the total mass for the galaxies in our sample.

By using the dynamical masses as an upper bound on the gas mass, and using the $\mathrm{H} \alpha$ line measurements and/or stellar population fits to infer star formation rates, we have examined the way the Lyman- $\alpha$ galaxies fall on the scaling relations for star formation. We conclude that they form stars more actively than the "normal" star forming galaxy population at comparable gas mass surface density. Their behavior is consistent with that observed in starburst galaxies, despite the typically smaller masses and sizes of the Lyman- $\alpha$ galaxy population.

The dynamical masses we infer remain 1-2 orders of magnitude below the characteristic dark halo masses inferred from the clustering of Lyman- $\alpha$ galaxies. Since the ratio of baryonic to dark mass should be globally uniform at around $14 \%$, we infer that a significant part of the baryonic matter associated with these halos has not yet accreted to the central kpc, where the active star formation is observed.

Finally, we examined densities of these objects. While hierarchical structure formation models suggest that small galaxies at $z \sim 2-3$ should become parts of early type galaxies or spiral bulges by $z=0$, the Lyman- $\alpha$ galaxies are of smaller size and comparable density to present day elliptical galaxies, and of comparable size but smaller density than present day spiral galaxy bulges. If the Lyman- $\alpha$ galaxies are to evolve into either, they must do so through dissipational merging. This is consistent with the picture of Lyman- $\alpha$ galaxies as young, starbursting galaxies, whose present properties and future evolution include a large role for gas physics. 


\section{Acknowledgments}

We thank the DARK Cosmology Centre and the Nordea Fonden in Copenhagen, Denmark, for hospitality during the completion of this work. We thank Ignacio Ferreras and Sune Toft for helpful discussions. This work has been supported by the US National Science Foundation through NSF grant AST-0808165. Based in part on observations obtained at the Gemini Observatory, which is operated by the Association of Universities for Research in Astronomy, Inc., under a cooperative agreement with the NSF on behalf of the Gemini partnership: the National Science Foundation (United States), the Science and Technology Facilities Council (United Kingdom), the National Research Council (Canada), CONICYT (Chile), the Australian Research Council (Australia), Ministério da Ciência, Tecnologia e Inovação (Brazil) and Ministerio de Ciencia, Tecnología e Innovación Productiva (Argentina). This work was supported in part by a NASA Keck PI Data Award, administered by the NASA Exoplanet Science Institute. Some data presented herein were obtained at the W. M. Keck Observatory from telescope time allocated to the National Aeronautics and Space Administration through the agency's scientific partnership with the California Institute of Technology and the University of California. The Observatory was made possible by the generous financial support of the W. M. Keck Foundation. The authors wish to recognize and acknowledge the very significant cultural role and reverence that the summit of Mauna

Kea has always had within the indigenous Hawaiian community. We are most fortunate to have the opportunity to conduct observations from this mountain.

\section{REFERENCES}

Bertin, E., \& Arnouts, S. 1996, A\&AS, 117, 393

Bezanson, R., van Dokkum, P. G., Tal, T., Marchesini, D., Kriek, M., Franx, M., \& Coppi, P. 2009, ApJ, 697, 1290

Bohlin, R. C., Savage, B. D., \& Drake, J. F. 1978, ApJ, 224, 132

Bond, N. A., Gawiser, E., Gronwall, C., Ciardullo, R., Altmann, M., \& Schawinski, K. 2009, ApJ, 705, 639

Capak, P., et al. 2007, ApJS, 172, 99

Cappellari, M., et al. 2006, MNRAS, 366, 1126

Daddi, E., et al. 2010, The Astrophysical Journal Letters, 714, L118 
Table 1: Basic observational properties of the sample.

\begin{tabular}{llllll} 
ID & $\mathrm{z}$ & $\sigma$ & $r_{e}(\mathrm{kpc})$ & SFR & Refs. $\stackrel{a}{-}$ \\
\hline LAE40844 & 3.1117 & 81 & 1.1 & 120 & 1 \\
LAE27878 & 3.11879 & 43 & 1.3 & 34 & 1 \\
HPS194 & 2.287 & 64.7 & 1.5 & 18 & 2 \\
HPS256 & 2.491 & 74.0 & 1.1 & 18 & 2 \\
COSMOS13636 & 2.1621 & 57 & 0.787 & 38 & 3 \\
COSMOS30679 & 2.19855 & 55 & 1.825 & 395 & 3 \\
CDFS3865 & 2.173 & 105 & 0.955 & 178 & 3 \\
CDFS6482 & 2.205 & 42 & 1.78 & 71 & 3 \\
LAE-COSMOS-47 & 2.24654 & 30 & 1.139 & 9.2 & 4
\end{tabular}

${ }^{a}$ References - 1. McLinden et al. (2011); Richardson et al. (2013, in prep); 2. Finkelstein et al. (2011); Song \& al (2013. in prep); 3. Hashimoto et al. (2012); Nakaiima et al. (2012); 4 . Nilsson et al. (2011)

Table 2. Best fitting SED model for each object.

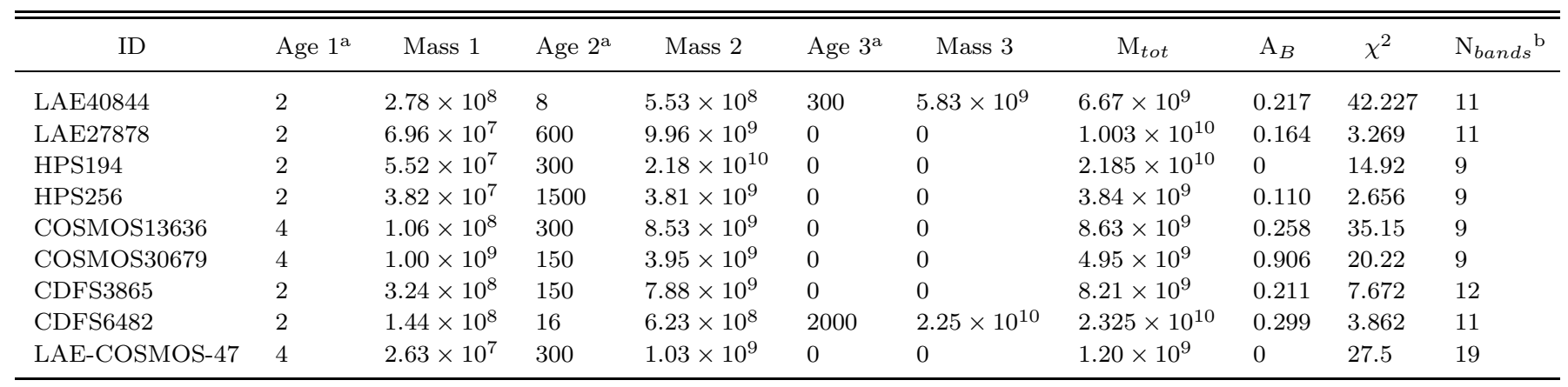

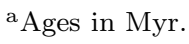

${ }^{\mathrm{b}}$ Number of photometric bands used in SED fitting. Wavelength coverage always extends at least from B band $(0.44 \mu m)$ to IRAC Channel $1(3.6 \mu m)$.
} 
Table 3. Range of SED fitting results for the sample.

\begin{tabular}{|c|c|c|c|c|c|c|c|c|c|}
\hline ID & $M_{-2 \sigma}$ & $\mathrm{M}_{*,-1 \sigma}$ & $\mathrm{M}_{*,+1 \sigma}$ & $\mathrm{M}_{*,+2 \sigma}$ & $A_{B,-2 \sigma}$ & $A_{B,-1 \sigma}$ & $A_{B, \text { best }}$ & $A_{B,+1 \sigma}$ & $A_{B,+2 \sigma}$ \\
\hline LAE40844 & $1.00 \times 10^{9}$ & $2.90 \times 10^{9}$ & $3.62 \times 10^{10}$ & $4.16 \times 10^{10}$ & 0.081 & 0.150 & 0.217 & 0.22 & 0.241 \\
\hline LAE27878 & $1.77 \times 10^{9}$ & $4.05 \times 10^{9}$ & $2.39 \times 10^{10}$ & $3.85 \times 10^{10}$ & 0 & 0 & 0.164 & 0.201 & 0.254 \\
\hline HPS256 & $1.03 \times 10^{8}$ & $1.87 \times 10^{9}$ & $1.20 \times 10^{10}$ & $2.16 \times 10^{10}$ & 0 & 0.009 & 0.110 & 0.199 & 0.298 \\
\hline COSMOS13636 & $2.61 \times 10^{9}$ & $4.49 \times 10^{9}$ & $1.17 \times 10^{10}$ & $2.20 \times 10^{10}$ & 0.083 & 0.161 & 0.258 & 0.291 & 0.320 \\
\hline COSMOS30679 & $1.00 \times 10^{9}$ & $1.75 \times 10^{9}$ & $1.28 \times 10^{10}$ & $2.82 \times 10^{10}$ & 0.718 & 0.831 & 0.906 & 0.991 & 1.141 \\
\hline LAE-COSMOS- 47 & $5.77 \times 10^{7}$ & $5.77 \times 10^{8}$ & $2.13 \times 10^{9}$ & $3.33 \times 10^{9}$ & 0 & 0 & 0 & 0.093 & 0.172 \\
\hline
\end{tabular}

Table 4: Masses and densities of the sample.

\begin{tabular}{lllll} 
ID & $\mathrm{M}_{d y n}$ & $\log \left(\bar{\rho}_{e}\right)$ & $\mathrm{M}_{*, \min }$ & $\mathrm{M}_{*, \max }$ \\
\hline LAE40844 & $6.7 \times 10^{9}$ & -22.39 & $2.90 \times 10^{9}$ & $3.62 \times 10^{10}$ \\
LAE27878 & $2.2 \times 10^{9}$ & -23.08 & $4.05 \times 10^{9}$ & $2.39 \times 10^{10}$ \\
HPS194 & $5.8 \times 10^{9}$ & -22.85 & $2.12 \times 10^{10}$ & $2.26 \times 10^{10}$ \\
HPS256 & $5.6 \times 10^{9}$ & -22.47 & $1.87 \times 10^{9}$ & $1.20 \times 10^{10}$ \\
COSMOS13636 & $2.4 \times 10^{9}$ & -22.40 & $4.49 \times 10^{9}$ & $1.17 \times 10^{10}$ \\
COSMOS30679 & $5.1 \times 10^{9}$ & -23.16 & $1.75 \times 10^{9}$ & $1.28 \times 10^{10}$ \\
CDFS3865 & $9.8 \times 10^{9}$ & -22.04 & $6.94 \times 10^{9}$ & $1.52 \times 10^{10}$ \\
CDFS6482 & $2.9 \times 10^{9}$ & -23.38 & $9.68 \times 10^{9}$ & $3.48 \times 10^{10}$ \\
LAE-COSMOS-47 & $9.5 \times 10^{8}$ & -23.28 & $5.77 \times 10^{8}$ & $2.13 \times 10^{9}$
\end{tabular}


Fazio, G. G., et al. 2004, ApJS, 154, 10

Finkelstein, S. L., Malhotra, S., Rhoads, J. E., Hathi, N. P., \& Pirzkal, N. 2009, MNRAS, 393,1174

Finkelstein, S. L., Rhoads, J. E., Malhotra, S., Pirzkal, N., \& Wang, J. 2007, ApJ, 660, 1023

Finkelstein, S. L., et al. 2011, ApJ, 729, 140

Franx, M., Illingworth, G., \& Heckman, T. 1989, AJ, 98, 538

Gawiser, E., et al. 2006, The Astrophysical Journal Supplement Series, 162, 1

Guaita, L., et al. 2010, ApJ, 714, 255

Hashimoto, T., Ouchi, M., Shimasaku, K., Ono, Y., Nakajima, K., Rauch, M., Lee, J., \& Okamura, S. 2012, ArXiv e-prints

Hathi, N. P., Malhotra, S., \& Rhoads, J. E. 2008, ApJ, 673, 686

Jedrzejewski, R. I. 1987, MNRAS, 226, 747

Jorgensen, I., Franx, M., \& Kjaergaard, P. 1996, MNRAS, 280, 167

Kassin, S. A., et al. 2007, ApJ, 660, L35

Kelson, D. D., Illingworth, G. D., van Dokkum, P. G., \& Franx, M. 2000, ApJ, 531, 184

Kovač, K., Somerville, R. S., Rhoads, J. E., Malhotra, S., \& Wang, J. 2007, ApJ, 668, 15

Leauthaud, A., et al. 2007, The Astrophysical Journal Supplement Series, 172, 219

Leitherer, C., et al. 1999, ApJS, 123, 3

MacArthur, L. A., Ellis, R. S., Treu, T., U, V., Bundy, K., \& Moran, S. 2008, ApJ, 680, 70

Madau, P. 1995, ApJ, 441, 18

Malhotra, S., Rhoads, J. E., Finkelstein, S. L., Hathi, N., Nilsson, K., McLinden, E., \& Pirzkal, N. 2012, ApJ, 750, L36

McCracken, H. J., et al. 2010, ApJ, 708, 202

McLinden, E., \& al. 2013, in prep

McLinden, E. M., et al. 2011, ApJ, 730, 136 
Meurer, G. R., Heckman, T. M., Lehnert, M. D., Leitherer, C., \& Lowenthal, J. 1997, AJ, 114,54

Nakajima, K., Ouchi, M., Shimasaku, K., Hashimoto, T., Ono, Y., \& Lee, J. C. 2012, ArXiv e-prints

Nilsson, K. K., Östlin, G., Møller, P., Möller-Nilsson, O., Tapken, C., Freudling, W., \& Fynbo, J. P. U. 2011, Astronomy \& Astrophysics, 529, 9

Partridge, R. B., \& Peebles, P. J. E. 1967, ApJ, 147, 868

Pei, Y. C. 1992, ApJ, 395, 130

Peletier, R. F., Davies, R. L., Illingworth, G. D., Davis, L. E., \& Cawson, M. 1990, AJ, 100, 1091

Pirzkal, N., Malhotra, S., Rhoads, J. E., \& Xu, C. 2007, ApJ, 667, 49

Richardson, M. L. A., McLinden, E. M., Malhotra, S., Rhoads, J. E., Hibon, P., \& Levesque, E. 2013 , in prep

Rix, H.-W., et al. 2004, ApJS, 152, 163

Song, M., \& al. 2013, in prep

Toft, S., Gallazzi, A., Zirm, A., Wold, M., Zibetti, S., Grillo, C., \& Man, A. 2012, ApJ, 754, 3

Tully, R. B., \& Fisher, J. R. 1977, A\&A, 54, 661

Twite, J. W., Conselice, C. J., Buitrago, F., Noeske, K., Weiner, B. J., Acosta-Pulido, J. A., \& Bauer, A. E. 2012, Monthly Notices of the Royal Astronomical Society, 420, 1061

Vernet, J., et al. 2011, A\&A, 536, A105 